\title{
Synchronized dynamic dose reconstruction
}

\author{
Dale W. Litzenberg, ${ }^{\text {a) }}$ Scott W. Hadley, Neelam Tyagi, James M. Balter, \\ Randall K. Ten Haken, and Indrin J. Chetty \\ University of Michigan, Ann Arbor, Michigan 48109
}

(Received 16 May 2006; revised 30 August 2006; accepted for publication 16 October 2006; published 14 December 2006)

Variations in target volume position between and during treatment fractions can lead to measurable differences in the dose distribution delivered to each patient. Current methods to estimate the ongoing cumulative delivered dose distribution make idealized assumptions about individual patient motion based on average motions observed in a population of patients. In the delivery of intensity modulated radiation therapy (IMRT) with a multi-leaf collimator (MLC), errors are introduced in both the implementation and delivery processes. In addition, target motion and MLC motion can lead to dosimetric errors from interplay effects. All of these effects may be of clinical importance. Here we present a method to compute delivered dose distributions for each treatment beam and fraction, which explicitly incorporates synchronized real-time patient motion data and real-time fluence and machine configuration data. This synchronized dynamic dose reconstruction method properly accounts for the two primary classes of errors that arise from delivering IMRT with an MLC: (a) Interplay errors between target volume motion and MLC motion, and (b) Implementation errors, such as dropped segments, dose over/under shoot, faulty leaf motors, tongue-andgroove effect, rounded leaf ends, and communications delays. These reconstructed dose fractions can then be combined to produce high-quality determinations of the dose distribution actually received to date, from which individualized adaptive treatment strategies can be determined. () 2007 American Association of Physicists in Medicine. [DOI: 10.1118/1.2388157]

Key words: Monte Carlo, delivered dose, intra-fraction motion, real-time tracking

\section{INTRODUCTION}

Two primary classes of errors arise from delivering intensity modulated radiation therapy (IMRT) with a multi-leaf collimator MLC: (a) Interplay errors between target volume motion and MLC motion, and (b) Implementation errors, such as dropped segments, dose over/under shoot, slow leaves, tongue-and-groove effect, rounded leaf ends, and communications delays. For purposes of this work, interplay errors are considered to be dosimetric errors that arise due to variations in the relative motions of the MLC leaves and the patient anatomy, from those included in the treatment planning process. It has been shown that interplay effects between moving MLC leaves, and the moving target volume, lead to dosimetric errors of over $100 \%$ in a given fraction. ${ }^{1}$ The magnitude of these errors depends on the relative orientation of the leaf and organ motion, the characteristic frequency of motion and their amplitudes. ${ }^{1}$ This effect has been shown experimentally in several intra-fraction motion studies. One study, ${ }^{2}$ of clinical head and neck cases, showed deviations as large as $20 \%$ compared to static measurements. In another phantom study, ${ }^{3}$ variations as large as $30 \%$ were observed in one fraction, and variations as large as $18 \%$ were observed with five super-imposed beams. Consequently, many possible scenarios can lead to measurable dosimetric errors in the implementation and delivery of IMRT.

The majority of IMRT treatments are delivered using a multi-leaf collimator. Leaf sequencing algorithms are intended to determine the leaf trajectories that will most accurately reproduce the desired fluence pattern during delivery. ${ }^{4-14}$ However, these algorithms have limitations and must make assumptions. For example, due to transmission, it is impossible to create a sequence that will deliver zero fluence to any given beamlet, which may lead to dosimetric errors up to $10 \%$, relative to the maximum beamlet dose. In addition, some algorithms do not correct for the tongue-andgroove effect, which may cause dosimetric errors of approximately $15 \%$. $^{12,15-19}$

It has also been shown that some leaf sequences may lead to dosimetric errors due to hardware and software delivery constraints. For example, communications delays in the MLC control system and inappropriate leaf position tolerance settings may lead to hundreds of beam interruptions. ${ }^{9}$ This has been shown to lead to $10 \%$ dose discrepancies and unpredictable delivery times. ${ }^{9,20}$ The communications delay also leads to dose overshoot on the first segment and undershoot on the last segment. ${ }^{21}$ The communications delay is related to the operating frequency of the MLC controller [20 Hz on the Varian Millennium MLC (Varian Medical Systems, Palo Alto, CA)] which determines the temporal resolution for sampling the leaf trajectories. Leaf sequencing algorithms that do not include these effects may create leaf sequences that attempt to deliver too many dose segments in a very short time. This can cause some dose segments to be omitted during delivery. ${ }^{22}$ There are also reports of leaves moving while dose is being delivered in step-and-shoot IMRT. $^{23}$ This is typically caused by a large leaf position tolerance and can lead to changes in dose at beamlet edges. 
In order to successfully individualize and refine a patient's treatment, a complete knowledge of the cumulative dose delivered to date is needed. Thus Dynamic Dose Reconstruction, based on synchronized real-time target volume and machine motion data, is needed to make dynamic treatment refinements as effective as initially envisioned. It is instructive to review some of the methods that have sought to include patient motion in treatment planning. Because the patient's anatomy varies from fraction to fraction, the cumulative dose after any given fraction is different from that determined during treatment planning on a static computed tomography (CT) patient model. Many techniques have been developed to estimate the cumulative dosedelivered-to-date for a given patient. ${ }^{24}$ These typically involve replanning and summing the patient's individual treatment fractions to account for positional variations in the target volume determined before each treatment fraction. In replanning these fractions, differing levels of sophistication are used in mapping the variable pre-fraction anatomy geometry back to the reference geometry used for the initial treatment planning. In some cases, the pre-treatment planning CT model is simply translated to correct for target volume shifts, while in other cases, image warping is used to map prefraction cone beam computed tomography (CB)CT models back to the pre-treatment planning $\mathrm{CT}$ reference geometry. $^{24,25}$

Intra-fraction motion is typically included via several computational methods. In some cases the anticipated breathing motion is convolved with the dose distribution, or time-weighted variations of the anticipated geometries are planned and summed. ${ }^{26-33}$ These idealized models of the intra-fraction motion assume that the amplitude, offset, frequency and functional form are constant for the entire course of therapy. ${ }^{3,34}$ In addition, values are typically chosen as average values for a population of patients instead of using values for that specific patient. Consequently, deviations in at least one of these parameters are likely to be common.

While these techniques are an improvement over conventional treatment planning on static CT models, they have several inherent limitations. Assumptions are made about the amplitude, frequency, and functional form of the motion so that probability distribution functions can be estimated. ${ }^{27,28,30,35-37}$ These are assumed to be constant for every fraction of treatment, though a recent study shows that there are significant variations in the mean position, amplitude and period from one breathing cycle to the next. ${ }^{38}$ Furthermore, in the case of dynamic IMRT delivery with a multi-leaf collimator (MLC), dose calculation algorithms generally assume that there are no interplay effects between the motion of the MLC leaves and the motion of the target volume. However, several groups are investigating real-time modification of leaf trajectories during dynamic IMRT delivery to compensate for target volume motion. ${ }^{2,39-41}$ This technique has the goal of significantly reducing interplay effects, without increasing treatment times as would happen with beam gating.

Studies have also been conducted to measure the effects of intra-fraction motion and their cumulative effect over
20-40 fractions. ${ }^{2,3}$ These experiments seek to test the results of simulations of idealized intra-fraction motion, ${ }^{34}$ and consequently, use the same simplifying assumptions about patient motion in the design of the experiments. These experiments use sinusoidal motions with $1 \mathrm{~cm}$ amplitude that begin at random or offset phases, with periods of 4-5 s. Because the expectation value of the sine function is zero, and the dose gradients at beam edges associated with threedimensional cathode ray tube (3DCRT) and IMRT are roughly $0.4 \mathrm{~cm}$, one expects the dose variations to become small when averaging over roughly five or more initial phases, as seen in the measurements ${ }^{3}$ and simulations. ${ }^{34,42,43}$ While the initial justification of using a sine function was that irregular respiratory motion of patients could always be decomposed into sine waves by Fourier methods, this detail has largely been overlooked. No simulations or measurements on the effects of nonideal intra-fraction motion were found in the literature, using motions that include drifts, changes in amplitude, or changes in period, as are commonly found in measured patient data.

While conformal delivery of high doses has been shown to be beneficial in many treatment sites, its effectiveness is limited by target motion, MLC motion interplay and implementation effects. Previous offline adaptive protocols suffered from the difficulty of obtaining patient specific information and the necessity of making simplifying statistical assumptions about the distribution of the measured variations in position. Likewise, current methods of including motion in treatment planning calculations make similar simplifying assumptions about the nature of the motion and ignore interplay and delivery effects that are assumed to average out over the course of treatment.

In a true accounting for inter- and intra-fraction motion and delivery interplay effects, dose accumulation techniques must account for real-time spatial and temporal changes in the intra-fraction motion of the patient and in the MLC leaves in a synchronized manner. Synchronizing real-time motion and fluence data will yield delivered dose reconstruction data that accurately reflect the following effects: interand intra-fraction setup errors and motion, motion interplay effects, temporal interplay effects during beam holds, tongue-and-groove effect, dropped segments, dose over- and undershoot, and leaf position errors. These effects will be accurately taken into account, without making simplifying assumptions about target volume motion or delivery effects. Consequently, by synchronizing this information to the beginning of the fraction, a complete real-time record of the fluence patterns delivered to the time-varying target volume position is obtained for every beam of each fraction. Thus, synchronized dynamic dose reconstruction is a tool that will allow cumulative deviations from the treatment plan to be assessed over the course of treatment, so that corrective action may be taken as necessary to ensure the prescribed dose distribution is delivered as accurately as possible.

\section{MATERIALS AND METHODS}

In the following sections, we will describe the real-time fluence and machine configuration data, and the real-time 
target volume tracking data necessary as input for the synchronized dynamic dose reconstruction method. The calculation method itself is then described, followed by the proofof-principle measurements and calculations.

\section{A. Real-time tracking of the treatment machine MLC}

In order to determine the cumulative dose delivered to the moving patient geometry, we must also know the shape of the fluence pattern delivered as a function of time over the course of each IMRT field delivered during a treatment fraction. On Varian treatment machines, this information is recorded in the DynaLog files. ${ }^{9,44-48}$ These files contain the leaf positions and beam state (on/off) at $50 \mathrm{~ms}$ intervals for a given IMRT treatment field.

Previous studies have validated the accuracy of the information contained in these files and found the reported leaf positions to agree with measurements to within the measurement uncertainty $(< \pm 0.1 \mathrm{~cm}$ at isocenter), and the delivered MU per segment to agree with measurements to within $2 \% .^{46,47}$ In addition, these studies have shown that the data in the DynaLog files accurately reflects deviations from idealized behavior, such as dropped segments, first segment dose overshoot and last segment dose undershoot, and leaf motion during step-and-shoot IMRT delivery. ${ }^{45}$

\section{B. Measuring real-time target volume motion with wireless transponders}

Several technologies exist for real-time tracking of intrafraction motion including ultrasound, fluoroscopy, and electromagnetic tracking. In this work, we used an electromagnetic tracking system (Calypso Medical Technologies, Inc.). The components, capabilities and accuracy of this system have been previously reported ${ }^{49-51}$ and are summarized here for convenience. The system uses three implantable wireless transponders, continuously tracked during therapy using an external electromagnetic array, to establish target position ten times per second. The system has been shown to have submillimeter accuracy throughout the active tracking volume, and readout precision ranging from $0.001 \mathrm{~cm}$ at $8 \mathrm{~cm}$ from the array, to $0.04 \mathrm{~cm}$ at $27 \mathrm{~cm}$ from the array. In-vault experiments demonstrated submillimeter accuracy in measuring known offsets of transponders in a phantom. Comparison of radiographic to electromagnetic localization, both in phantoms and in patients, demonstrated that the system is capable of accuracy similar to that from orthogonal diagnostic radiographs. Experiments to test the accuracy of moving transponders showed that the $3 \mathrm{D}$ rms positional error was less than $0.054 \mathrm{~cm}$ for velocities up to $3 \mathrm{~cm} / \mathrm{s}$.

\section{Real-time target volume tracking data from initial clinical studies}

Initial clinical studies of the Calypso system were conducted to evaluate positional stability and implant experience with implanted transponders. ${ }^{50}$ Inter-transponder distances were typically stable by day four postimplant but at the latest by day 14 , when the CT planning simulation was performed.
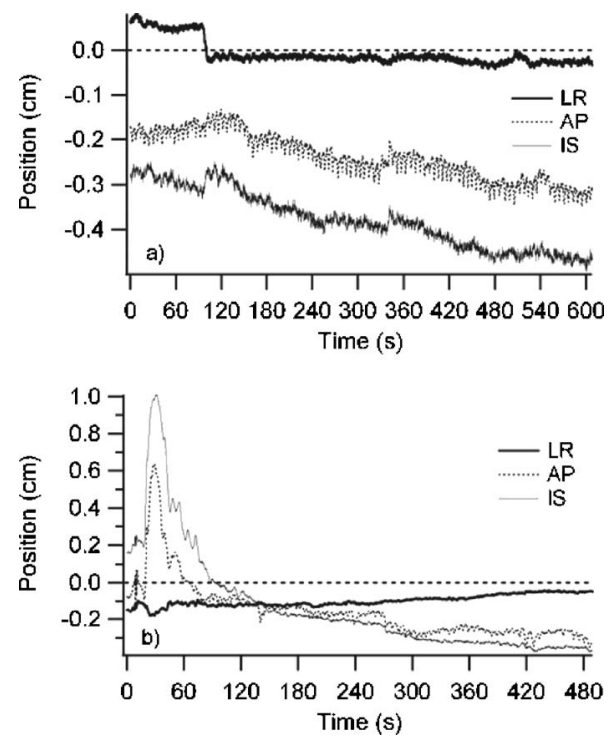

FIG. 1. Data from two patients who were monitored at $10 \mathrm{~Hz}$, for $8 \mathrm{~min}$ each, using the Calypso ${ }^{\circledR}$ 4D localization system. Figure (a) shows a maximum deviation in any direction of less than $0.3 \mathrm{~cm}$, which is typical for nine of the 11 patients monitored, while (b) shows much larger deviations, which were seen in two patients.

The mean standard deviation of inter-transponder distances from day 14 through fraction 20 in 20 patients was $0.08 \mathrm{~cm}$ (range $0.02-0.19 \mathrm{~cm}$ ).

In initial clinical studies, 11 of 20 patients participated in an $8 \mathrm{~min}$ session in which the transponders were tracked continuously at $10 \mathrm{~Hz}$. Intra-treatment movements were observed on time scales inconsistent with breathing (i.e., not periodic with a roughly 4-5 s period) and with magnitudes ranging up to $1.3 \mathrm{~cm}$. Figure 1 shows two example plots of prostate motion monitored at $10 \mathrm{~Hz}$ using this prototype system. Figure 1(a) shows a slow, continuous trend in motion along these axes over the entire observation time, while the Fig. 1(b) shows movement of the prostate in the anteriorposterior and inferior-superior directions early during the monitoring period. High frequency components in these plots indicate the quiet breathing pattern of these supine patients. Overall, after initial positioning, the average prostate movement seen across 11 patients during the observation time after initial positioning ranged from 0.13 to $0.58 \mathrm{~cm}$ (average $0.31 \mathrm{~cm}$ ), with maximum per-patient temporal excursions from 0.19 up to $1.20 \mathrm{~cm}$.

This system is well suited for measuring translations and rotations about the center of the implanted Beacons and in rigid geometries. The use of this technology, in conjunction with cone beam CT and other technologies, in deforming geometries is briefly discussed in Section IV D. The TimeDependent Geometric Patient Model.

\section{Monte Carlo simulations}

In this section, we present the synchronized dynamic dose reconstruction method, followed by the source model and MLC model implemented within the Monte Carlo algorithm. 


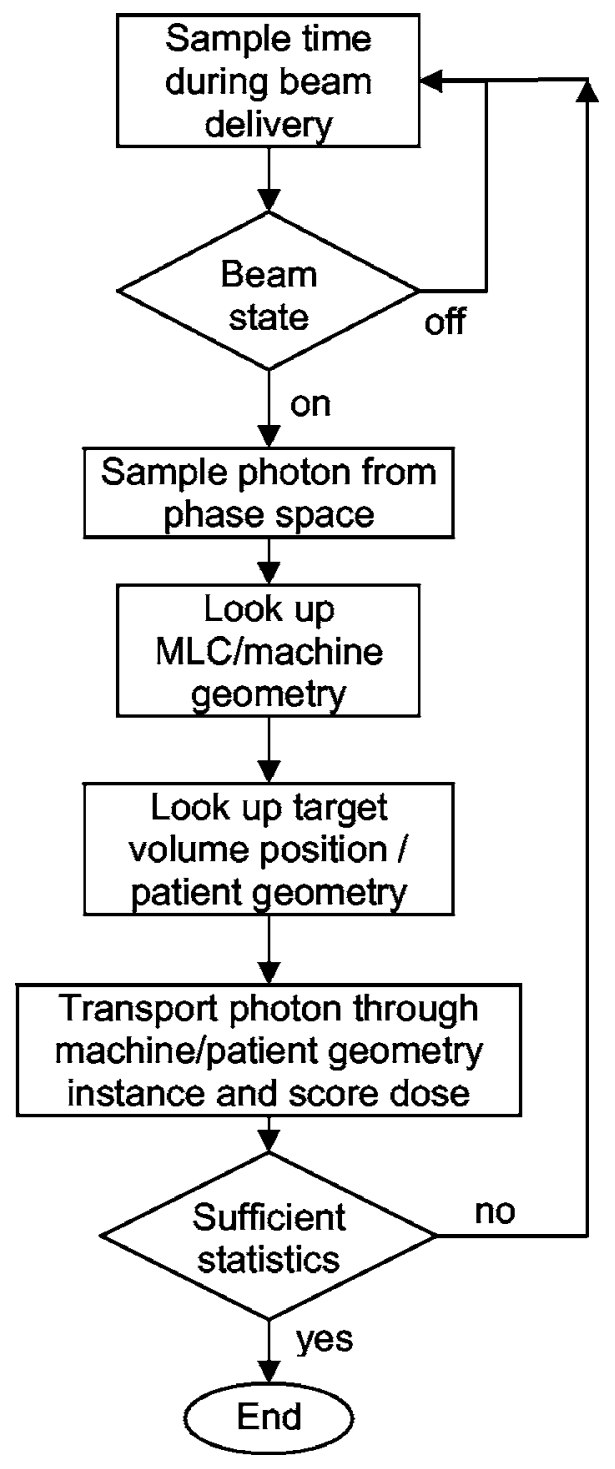

FIG. 2. Flow chart for the SDDR method implemented in the DPM Monte Carlo dose calculation algorithm.

\section{Synchronized dynamic dose reconstruction method}

This method is implemented using the dose planning method (DPM) Monte Carlo code developed by Bielajew and collaborators ${ }^{52}$ for radiation therapy treatment planning calculations. The code has been validated, ${ }^{53-56}$ integrated within our in-house treatment planning system, UMPlan, ${ }^{57,58}$ and preliminary clinical treatment planning studies conducted. ${ }^{28,57}$ The method is illustrated in Fig. 2 and implemented as follows. A time (not a monitor unit fraction) during a beam delivery was randomly generated from a uniform distribution and compared to the "beam-on" flag at the corresponding time in the DynaLog files. If the beam was on, a photon was sampled from a pre-calculated phase-space plane located above the jaws. The MLC leaf positions at that time are also determined from the DynaLog files, and the phantom position (or target volume position) determined from the measured motion profile. The photon is then propagated through that instance of the MLC and patient geometry. This process is repeated until the desired level of dosimetric uncertainty is achieved.

The uniform time sampling technique allows this algorithm to account for periods when the beam is off, and still advance target volume motion without depositing dose. This allows spatial and temporal interplay effects to be included in the dose calculation, as well as all delivery related issues recorded in the DynaLog files.

\section{Source model}

A source model of a Varian 2100EX linear accelerator and the 120-leaf multi-leaf collimator (MLC) has been developed for simulations in this study. ${ }^{59}$ The model is based on BEAMnrc $^{60}$ simulations of the patient-independent components, the target, primary collimator, flattening filter, ion chamber, and mirror. The DPM MC code is used for the patient-dependent calculation, i.e., the MLC, jaws and patient. ${ }^{59}$ As described in a previous publication. ${ }^{59}$ the MLC leaf model accounts for details in the MLC design, such as a single focus, tongue-and-groove geometry, the rounded leaf tip the and the air spaces between adjacent leaves. The model has been benchmarked against measurements under a variety of circumstances and been shown to be in good agreement with measurements under stringent test conditions, such as the closed leaf leakage patterns. ${ }^{59}$

\section{E. Phantom Studies and Simulations}

In this section, the phantom configuration and IMRT beams used are described, followed by a brief description of the programmable translation stage and motion profiles used during measurements. This is followed by a description of the analysis used for film measurements and synchronized dynamic dose reconstruction (SDDR) simulation results, and the methods used for determining uncertainties.

\section{Phantom configuration}

To demonstrate the concept of the SDDR method, measurements were conducted and compared to simulations. In these measurements, 14 clinical IMRT beams from two patients on a urethral sparing protocol were delivered to stationary and moving Solid Water slab phantoms. Measurements were made with film at $5 \mathrm{~cm}$ depth in a $15 \times 40$ $\times 40 \mathrm{~cm}^{3}$ Solid Water phantom with an source to surface distance (SSD) of $95 \mathrm{~cm}$. Kodak, XV, redipak film, was chosen because the dynamic range of the film was well suited to the range of beamlet doses, between 5 and $100 \mathrm{cGy}$, found in these urethural-sparing prostate IMRT fields. All fields were measured individually and found to be within the dynamic range of the XV film. All films were from a single production batch. Absolute dosimetry was determined by measuring an $H \& D$ curve at 16 doses spanning the dynamic range of the film under standard reference conditions $(10 \mathrm{~cm} \times 10 \mathrm{~cm}$ field at $10 \mathrm{~cm}$ depth, $90 \mathrm{~cm}$ SSD in a $30 \mathrm{~cm} \times 30 \mathrm{~cm}$ $\times 20 \mathrm{~cm}$ water equivalent phantom). 


\section{Translation stage and motion profiles}

The phantom was placed on a programmable translation stage (driven by a servo motor with feedback) capable of reproducing arbitrary motion profiles with $<=0.05 \mathrm{~cm}$ average positional error for amplitudes up to $6 \mathrm{~cm}$, velocities up to $8 \mathrm{~cm} / \mathrm{s}$, and accelerations up to $10 \mathrm{~cm} / \mathrm{s}^{2}$ with a phantom up to $25 \mathrm{Kg} .{ }^{61}$ Motion is synchronized to within $2 \mathrm{~ms}$ of the beginning of the beam by triggering an input/output port on the motion controller using a modified signal from the beam target in the accelerator.

In addition to film measurements in stationary phantoms, measurements were made with the phantom following two different motion profiles. The first profile is a typical idealized cosine function with the form

$$
x(t)=\cos ^{4}(\pi t / 4),
$$

which has a $1 \mathrm{~cm}$ peak-to-peak amplitude and a $4 \mathrm{~s}$ period. The second motion profile was the clinically measured inferior-superior motion of the prostate, as shown in Fig. 1(b), as measured with the Calypso system. This profile will be referred to as the measured motion, and indicated with a subscript " $m$ ", in film measurements, $F_{m}$, and simulation results, $S_{m}$. The SDDR method was used to simulate the delivered dose in the plane of the film for the stationary and clinically measured motions, while standard Monte Carlo fluence convolution dose calculation was used to estimate dose to the film plane for measurements with the idealized breathing motion.

\section{Analysis of film and simulation results}

The measured and simulated planar dose distributions were then compared (as described below) to characterize the difference between static dose distributions, synchronized dynamic dose distributions with measured patient motion, and fluence convolution with idealized patient motion. Film measurements were spatially calibrated using a Lucite template with pinholes placed at $\pm 11 \mathrm{~cm}$ longitudinally and $\pm 12 \mathrm{~cm}$ laterally. The template was positioned on the film to coincide with the cross hairs of the light field, and pin holes were then made in the film. The films were digitized and the pinholes used to set the spatial scale, correct film rotations, and set isocenter coordinates. The images were then interpolated (bilinearly) to a common grid and subtracted. This technique was used to compare dose distributions that were not expected to be identical (static vs moving distributions). A second method was used to compare dose distributions that were expected to be the same. In this method, the film measurements were cropped to eliminate the pin holes, and an image registration algorithm ${ }^{62}$ run to find the relative spatial alignment (including translations and rotations) that minimized the root-mean-square (rms) of the dose differences.

Variations between compared dose distributions were quantified by finding the mean and standard deviation of the differences in the smallest rectangular area around the dose difference distribution that excluded only background values [as shown in Fig. 3(g)]. Consequently, the area of the rectangle for image registration may have been larger than the smallest rectangle around the static fluence pattern if the measurement was made in a moving phantom.

Table I summarizes the dose distributions, $D_{1}$ and $D_{2}$, that were compared $\left(\Delta D=D_{1}-D_{2}\right)$. Simulations in moving phantoms $\left[S_{m}\right.$, simulation of dose delivered with phantom moving with the Calypso measured clinical motion profile, $S_{c}$, simulation of dose delivered with phantom moving under idealized cosine motion given in Eq. (1)] were compared to the simulation in a static phantom, $S_{s}$. Film measurements in moving phantoms, $F_{m}$ and $F_{c}$, respectively, were compared to the first film measurement in a static phantom, $F_{s}$. Simulations were compared to the corresponding film measurements, and repeat films of static beams, $F_{s 2}$ and $F_{s 3}$, were compared to estimate uncertainties due to alignment technique and film measurements.

\section{F. Determination of uncertainties}

In the phantom studies simulated here, sufficient histories were run such that the typical uncertainty within the field was $1.4 \%$ at the maximum dose point, and was better than $2 \%$ in the average dose to the volume receiving greater than $50 \%$ of the maximum dose.

To estimate the uncertainties associated with the film measurements, all seven beams of one of the two cases were measured in a static phantom three times: twice on the same treatment machine and again on a second machine. In addition, film measurements involving motion were repeated twice for each of the seven beams in one of the two cases. Static films 1, 2, and 3 for each beam were then compared to each other using both alignment techniques to quantify the uncertainties associated with each technique.

\section{RESULTS}

In this section, we first present comparisons between SDDR simulations and film measurement undergoing the same motion. This is followed by the measured and reconstructed dosimetric differences between a static phantom and a moving phantom. The uncertainties due to film measurements, alignment techniques and simulation results are then presented.

\section{A. SDDR simulations versus film measurements}

A set of sample comparisons is shown first for one beam, then followed by the results of all comparisons in tabular form. Figures 3(a)-3(c) show the Monte Carlo simulations in the static phantom, and the phantom moving with a cosine function and clinically measured motions in the top row, respectively. The second row, Figs. 3(d)-3(f), shows the corresponding film measurements, while the third row, Figs. $3(\mathrm{~g})-3(\mathrm{i})$, shows the difference between simulations and films. The mean and standard deviations of the differences are also shown on the difference image. The mean and standard deviations for all SDDR-to-film comparisons are shown in columns 1-3 (SDDR vs film) of Table II (case 1), and Table III (case 2), for static, $s$, cosine, $c$, and measured, $m$, motion profiles. In general, all average difference values 


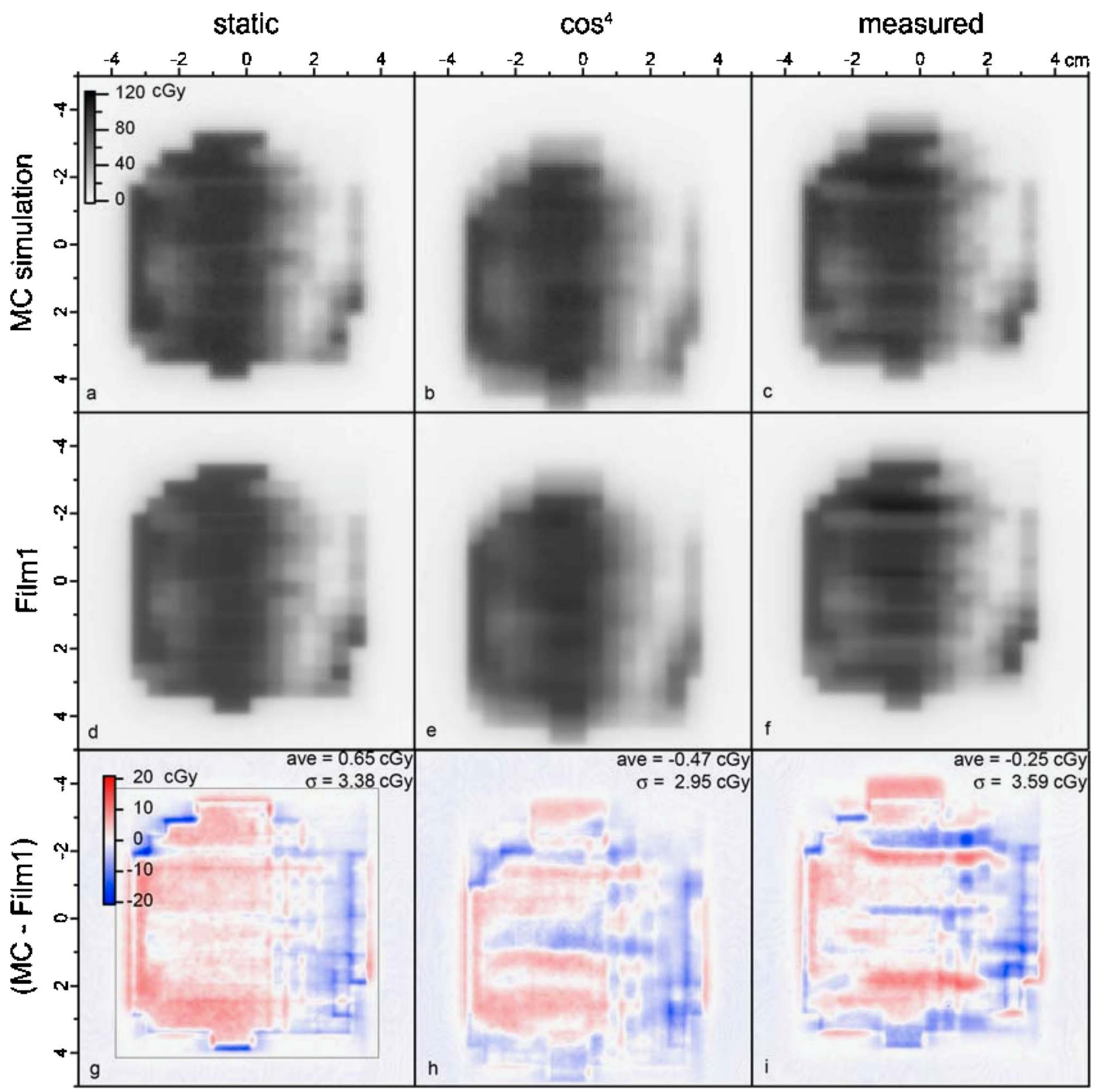

FIG. 3. Monte Carlo simulations in the static phantom, and the phantom moving with a cosine function [Eq. (1)] and clinically measured motions in the top row. The second row shows the corresponding film measurements, while the third row shows the difference between simulations and films. The mean and standard deviations of the differences are also shown for the smallest rectangular region containing dose differences above background, as shown in the bottom left.

given in Tables II and III should be zero, regardless of motion profile, since the same dose is delivered to the two distributions being compared.

The average value of all 21 dosimetric differences between films and simulations in columns 1-3 (seven beams, each measured and simulated in static, cosine, and clinically measured motion profiles) for case 1 is $0.01 \mathrm{cGy}$, while for case 2 it is $0.88 \mathrm{cGy}$, indicating very good agreement between the measured and simulated absolute doses. The standard deviation of differences between the measured distributions and the simulated distributions is typically $3-4 \mathrm{cGy}$ in both static and moving phantoms. While the typical differ- ence is generally $<|2|$ cGy across the field, the standard deviation is strongly influenced by small areas of disagreement at leaf edges.

\section{B. Simulated and measured deviations from static dose distributions}

In clinical practice, the difference between a statically planned and delivered case, versus the dose distribution actually received in the presence of motion, is the primary concern. The dose differences caused by idealized cosine motion, compared to a static phantom, are shown in Fig. 4(a) 
TABLE I. Illustration of comparisons $\left(\Delta D=D_{1}-D_{2}\right)$, where the first letter denotes simulation, $S$, or film measurement, $F$, and the second letter denotes either static phantom, $s$, a phantom moving with a cosine motion, $c$, or a phantom moving with a clinically measured motion, $m$. Static films were measured three times on two machines, while motion measurements were made twice each on the same machine.

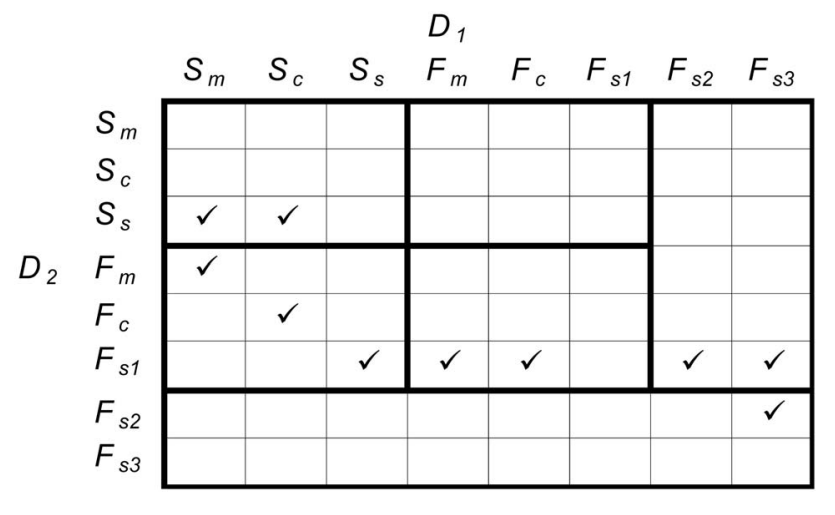

(SDDR) and Fig. 4(c) (film measurement). The dose differences caused by the clinically measured motion, compared to a static phantom, are shown in Fig. 4(b) (SDDR) and Fig. 4(d) (film measurement). The mean and standard deviations for each of the seven beams are summarized in columns four and five of Tables II and III, for film measurements (cosine minus static, and measured minus static), and in columns six and seven for SDDR reconstructions. The average value of differences between static and moving films is about $0.7 \mathrm{cGy}$, while it is only $0.03 \mathrm{cGy}$ for simulation comparisons. The standard deviation of differences between cosine and static motions was 18.80 and $18.36 \mathrm{cGy}$ for film and SDDR reconstructions, respectively, while between clinically measured and static motions they were 10.35 and $9.55 \mathrm{cGy}$ for film and SDDR reconstructions, respectively. Note that the simulation results are expected to have perfect registration, and therefore lower standard deviations of the differences.

\section{Uncertainty due to film alignment technique}

To compare the above dose distributions between moving and static phantoms, the films must be spatially calibrated using the pinholes made in the film. (Image registration cannot be used here, as it was for the comparisons in Fig. 3, because the different motion profiles make the dose distributions different.) This leads to slight misalignments, resulting

TABLE II. Average and standard deviations for all comparisons in case 1. Differences between SDDR and film are shown in columns 1-3 (SDDR vs Film), for static, $s$, cosine, $c$, and measured, $m$, motion profiles. Columns 4 and 5 show differences between moving and static phantoms for film measurements, $F$, (cosine minus static film 1, and measured minus static film 1), and the corresponding SDDR comparisons, $S$, are shown in columns 6 and 7 . Columns $8-10$ show the differences between repeat films for all beams and motion cases using an intensity-based image registration technique, while the same differences are shown in columns 11-13 for a pinhole-based transformation. Columns 14-17 show differences between repeat static films measured on different machines with different alignment techniques.

\begin{tabular}{|c|c|c|c|c|c|c|c|c|c|c|c|c|c|c|c|c|c|}
\hline \multicolumn{18}{|c|}{ Average differences (cGy) } \\
\hline Column & 1 & 2 & 3 & 4 & 5 & 6 & 7 & 8 & 9 & 10 & 11 & 12 & 13 & 14 & 15 & 16 & 17 \\
\hline \multirow[b]{2}{*}{ Beam } & \multirow[b]{2}{*}{$\mathrm{S}$} & \multicolumn{3}{|c|}{ SDDR vs Film } & \multicolumn{4}{|c|}{ Motion vs Static } & \multicolumn{3}{|c|}{$(\mathrm{F} 2-\mathrm{F} 1) \mathrm{R}$} & \multicolumn{3}{|c|}{$(\mathrm{F} 2-\mathrm{F} 1) \mathrm{T}$} & \multicolumn{2}{|c|}{ (Fs3-Fs1) } & \multirow{2}{*}{$\frac{(\mathrm{Fs} 3-\mathrm{Fs} 2)}{\mathrm{T}}$} \\
\hline & & $\mathrm{c}$ & $\mathrm{m}$ & $\mathrm{Fc}-\mathrm{Fs} 1$ & Fm-Fs 1 & Sc-Ss & Sm-Ss & $\mathrm{s}$ & $\mathrm{c}$ & $\mathrm{m}$ & s & $\mathrm{c}$ & $\mathrm{m}$ & $\mathrm{R}$ & $\mathrm{T}$ & $\mathrm{R}$ & \\
\hline 1 & 0.10 & -0.42 & 0.31 & 0.72 & 0.83 & 0.37 & 0.23 & -0.67 & -0.38 & 0.15 & -0.67 & -0.40 & 0.12 & 1.86 & 1.90 & 1.22 & -1.26 \\
\hline 2 & 0.44 & -0.64 & -0.04 & 0.85 & 0.32 & -0.17 & -0.12 & -0.30 & -0.31 & 0.33 & -0.33 & -0.31 & 0.34 & 0.59 & 0.54 & 0.86 & 0.86 \\
\hline 3 & -0.67 & -1.10 & -0.72 & 0.45 & 0.30 & -0.03 & 0.19 & -0.77 & -0.36 & -0.15 & -0.78 & -0.36 & -0.21 & 0.06 & 0.04 & 0.81 & 0.79 \\
\hline 4 & 0.65 & -0.47 & -0.25 & 1.14 & 0.78 & -0.02 & -0.04 & -0.03 & -0.33 & -0.51 & -0.10 & -0.38 & -0.54 & 0.55 & 0.48 & 0.60 & 0.58 \\
\hline 5 & 0.87 & 0.22 & -0.61 & 0.39 & 1.45 & -0.18 & 0.10 & -0.62 & -0.49 & -1.19 & -0.62 & -0.48 & -1.17 & -0.05 & -0.10 & 0.58 & 0.52 \\
\hline 6 & 0.66 & 0.26 & 0.04 & 0.43 & 0.84 & 0.20 & 0.22 & -0.15 & -0.71 & -0.11 & -0.18 & -0.68 & -0.19 & 0.15 & 0.58 & 0.73 & 0.74 \\
\hline 7 & 1.00 & 0.29 & 0.12 & 0.62 & 0.87 & -0.05 & 0.05 & -0.74 & -0.51 & -0.28 & -0.71 & -0.51 & -0.30 & 1.24 & 1.22 & 1.98 & 1.96 \\
\hline ave & 0.44 & -0.27 & -0.16 & 0.66 & 0.77 & 0.02 & 0.09 & -0.47 & -0.44 & -0.25 & -0.49 & -0.45 & -0.28 & 0.63 & 0.67 & 0.97 & 0.60 \\
\hline \multicolumn{18}{|c|}{ Standard Deviation of Differences (cGy) } \\
\hline \multirow[t]{2}{*}{ Column } & 1 & 2 & 3 & 4 & 5 & 6 & 7 & 8 & 9 & 10 & 11 & 12 & 13 & 14 & 15 & 16 & 17 \\
\hline & \multicolumn{3}{|c|}{ SDDR vs Film } & \multicolumn{4}{|c|}{ Motion vs Static } & \multicolumn{3}{|c|}{$(\mathrm{F} 2-\mathrm{F} 1) \mathrm{R}$} & \multicolumn{3}{|c|}{$(\mathrm{F} 2-\mathrm{F} 1) \mathrm{T}$} & \multicolumn{2}{|c|}{ (Fs3-Fs1) } & \multicolumn{2}{|c|}{ (Fs3-Fs2) } \\
\hline Beam & $\mathrm{s}$ & $\mathrm{c}$ & $\mathrm{m}$ & Fc-Fs1 & Fm-Fs 1 & Sc-Ss & Sm-Ss & $\mathrm{s}$ & $\mathrm{c}$ & $\mathrm{m}$ & $\mathrm{s}$ & $\mathrm{c}$ & $\mathrm{m}$ & $\mathrm{R}$ & $\mathrm{T}$ & $\mathrm{R}$ & $\mathrm{T}$ \\
\hline 1 & 3.61 & 2.62 & 3.91 & 17.66 & 11.72 & 18.75 & 9.95 & 1.99 & 1.05 & 1.42 & 4.53 & 2.05 & 2.84 & 2.15 & 2.60 & 1.24 & 4.30 \\
\hline 2 & 3.78 & 2.91 & 3.80 & 19.82 & 9.06 & 19.59 & 9.40 & 1.59 & 1.01 & 2.37 & 5.74 & 2.39 & 4.13 & 1.40 & 6.99 & 1.77 & 6.18 \\
\hline 3 & 3.82 & 3.65 & 3.52 & 20.31 & 10.38 & 17.96 & 8.94 & 1.77 & 1.05 & 1.10 & 9.09 & 5.18 & 6.91 & 0.94 & 14.75 & 1.66 & 10.83 \\
\hline 4 & 3.38 & 2.95 & 3.59 & 16.36 & 9.57 & 17.32 & 7.98 & 1.54 & 1.38 & 0.96 & 5.52 & 4.15 & 4.02 & 1.25 & 7.06 & 1.28 & 4.73 \\
\hline 5 & 3.68 & 3.27 & 4.34 & 18.99 & 10.15 & 17.54 & 9.44 & 1.50 & 1.60 & 1.20 & 7.15 & 4.28 & 7.68 & 1.13 & 4.71 & 1.09 & 4.38 \\
\hline 6 & 3.21 & 2.89 & 3.34 & 18.95 & 10.88 & 17.51 & 9.42 & 1.93 & 1.28 & 0.95 & 6.26 & 2.58 & 4.99 & 1.94 & 5.75 & 1.61 & 2.07 \\
\hline 7 & 3.52 & 2.85 & 3.65 & 19.20 & 10.49 & 19.67 & 11.35 & 1.62 & 0.89 & 1.25 & 4.07 & 1.57 & 3.19 & 1.55 & 4.54 & 1.07 & 1.80 \\
\hline RMS $\sigma$ & 3.58 & 3.04 & 3.75 & 18.80 & 10.35 & 18.36 & 9.55 & 1.71 & 1.20 & 1.40 & 6.25 & 3.41 & 5.12 & 1.53 & 7.55 & 1.41 & 4.90 \\
\hline
\end{tabular}


TABLE III. Average and standard deviations for all comparisons in case 2.

\begin{tabular}{|c|c|c|c|c|c|c|c|}
\hline \multicolumn{8}{|c|}{ Average Differences (cGy) } \\
\hline Column & 1 & 2 & 3 & 4 & 5 & 6 & 7 \\
\hline \multirow[b]{2}{*}{ Beam } & \multicolumn{3}{|c|}{ SDDR vs Film } & \multicolumn{4}{|c|}{ Motion vs Static } \\
\hline & s & $\mathrm{c}$ & $\mathrm{m}$ & Fc-Fs1 & Fm-Fs1 & Sc-Ss & Sm-Ss \\
\hline 8 & 0.56 & 0.31 & -0.44 & 0.41 & 1.23 & -0.01 & 0.17 \\
\hline 9 & 1.55 & 0.97 & 0.21 & 0.56 & 1.32 & 0.04 & 0.09 \\
\hline 10 & 1.80 & 0.79 & 0.64 & 0.53 & 0.87 & -0.12 & -0.09 \\
\hline 11 & 1.07 & 0.76 & -0.14 & 0.33 & 1.19 & 0.17 & 0.04 \\
\hline 12 & 1.71 & 0.83 & -0.16 & 0.59 & 1.65 & -0.12 & -0.12 \\
\hline 13 & 2.78 & 1.91 & 0.22 & 0.76 & 2.42 & 0.12 & -0.01 \\
\hline 14 & 2.21 & 1.01 & -0.13 & 0.97 & 2.09 & -0.01 & -0.14 \\
\hline ave & 1.67 & 0.94 & 0.03 & 0.59 & 1.54 & 0.01 & -0.01 \\
\hline
\end{tabular}

Standard Deviation of Differences (cGy)

\begin{tabular}{|c|c|c|c|c|c|c|c|}
\hline \multirow[t]{2}{*}{ Column } & 1 & 2 & 3 & 4 & 5 & 6 & 7 \\
\hline & \multicolumn{3}{|c|}{ SDDR vs Film } & \multicolumn{4}{|c|}{ Motion vs Static } \\
\hline Beam & $\mathrm{s}$ & c & $\mathrm{m}$ & Fc-Fs 1 & Fm-Fs1 & Sc-Ss & $\mathrm{Sm}-\mathrm{Ss}$ \\
\hline ave & 1.67 & 0.94 & 0.03 & 0.59 & 1.54 & 0.01 & -0.01 \\
\hline 8 & 2.60 & 2.40 & 3.10 & 14.32 & 8.34 & 13.43 & 6.85 \\
\hline 9 & 3.63 & 2.72 & 3.17 & 16.02 & 9.45 & 7.38 & 19.95 \\
\hline 10 & 3.47 & 2.78 & 3.46 & 16.52 & 11.90 & 17.00 & 11.22 \\
\hline 11 & 3.25 & 2.42 & 3.30 & 16.33 & 8.01 & 16.44 & 8.28 \\
\hline 12 & 3.24 & 2.41 & 3.15 & 12.38 & 10.64 & 15.92 & 7.98 \\
\hline 13 & 4.24 & 3.54 & 3.97 & 18.69 & 8.68 & 18.94 & 9.80 \\
\hline 14 & 3.57 & 3.06 & 3.52 & 17.10 & 9.42 & 18.36 & 8.83 \\
\hline RMS $\sigma$ & 3.46 & 2.79 & 3.39 & 16.02 & 9.57 & 15.78 & 11.19 \\
\hline
\end{tabular}

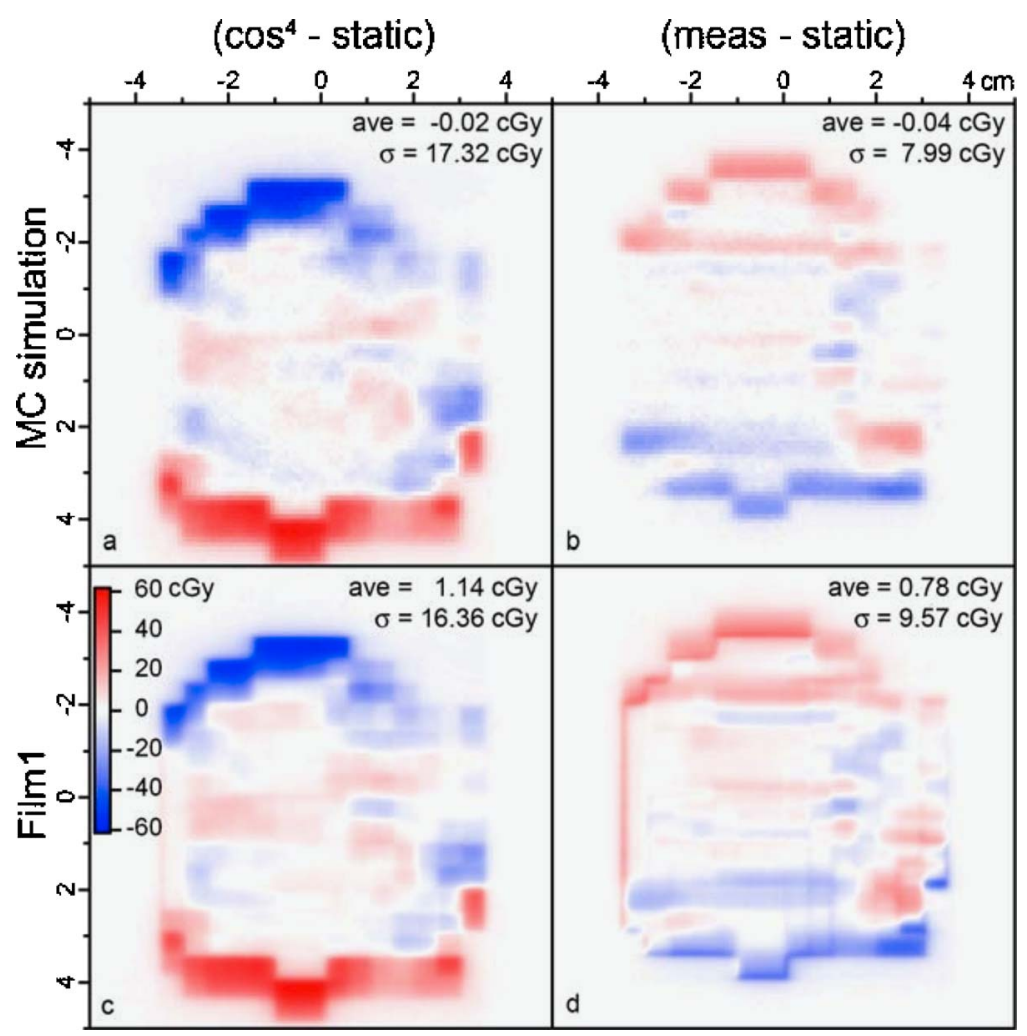

in larger dose differences than typically observed when using intensity based image registration. To characterize the differences between these two registration techniques, repeat film measurements were aligned using both techniques. Figure 5 shows the typical difference between two static films of the same beam that were aligned using both pinholes from a template and image registration. The mean and standard deviation of the differences between film 2 and film 1, using the intensity-based registration technique, are shown in columns 8-10 of Table II, while the same results using the pinhole based transformation are shown in columns 11-13. The average differences are all about -0.4 cGy regardless of registration technique or motion profile. However, the standard deviations for the image registration technique range between 1.2 and $1.71 \mathrm{cGy}$, depending on the motion profile, while for the pinhole transformation technique they range between 3.41 and $6.25 \mathrm{cGy}$. The standard deviations within each registration technique increase from cosine motion, to clinically measured to static motions, as would be expected as the distributions become less blurred by the motion. If we assume that the standard deviation of the pinhole registration is the quadratic sum of an intensity-based component, quantified by the image registration technique and a misalignment-based component, we see that the variations due to small misalignments dominate over intensity-based differences.

\section{Uncertainty in SDDR Monte Carlo simulations}

Figure 6 shows the uncertainty in the simulation of a static phantom. Uncertainties within the field range between
FIG. 4. The difference between each motion case and the static case, for both films and simulations. 


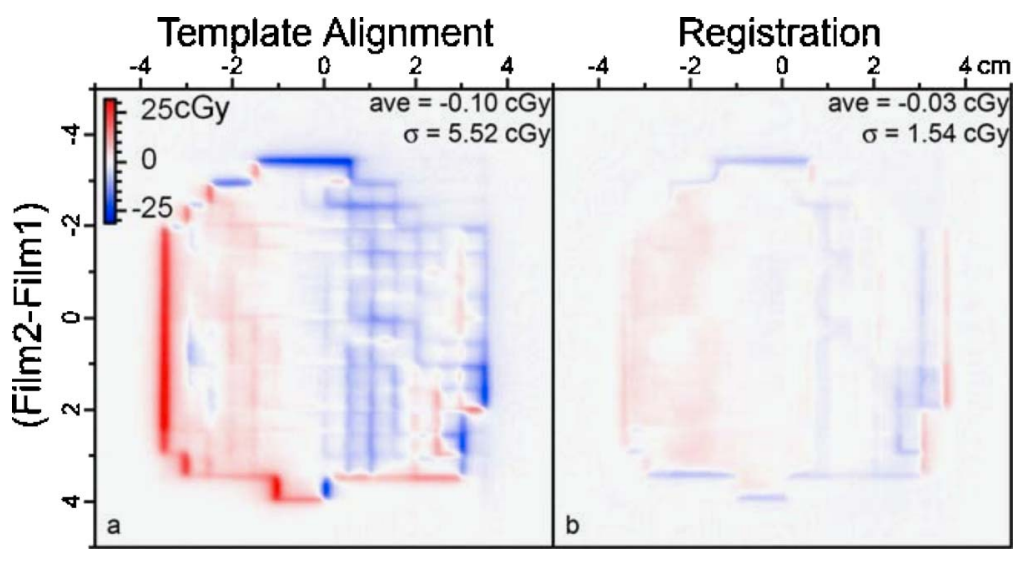

FIG. 5. The typical difference between two static films of the same beam which were aligned using both pinholes from a template and image registration.
$1.4 \%$ and $2.0 \%$ of the maximum dose, which corresponds to roughly $1.2-1.8 \mathrm{cGy}$. This is comparable to the standard deviation of differences found between repeat film measurements $(1.2-1.7 \mathrm{cGy})$ for all seven beams and all three motion profiles in case 1 when using intensity-based image registration.

\section{DISCUSSION}

In this section, the agreement between the film measurements and the simulations is discussed first, followed by the magnitude of the dosimetric errors due to motion as quantified by measurements and simulations. The uncertainty in the results is discussed based on repeated film measurements and the registration technique used for making comparisons. And finally, the applicability of the method in nonrigid patient geometries is discussed.

\section{A. Comparison of film measurements and simulations}

As seen in Fig. 3, the measured and simulated dose distributions in static phantoms, and phantoms moving with the cosine function [Eq. (1)] and clinically measured motion profiles, are generally well reproduced by the SDDA method. Features of the dose distributions measured with film are also

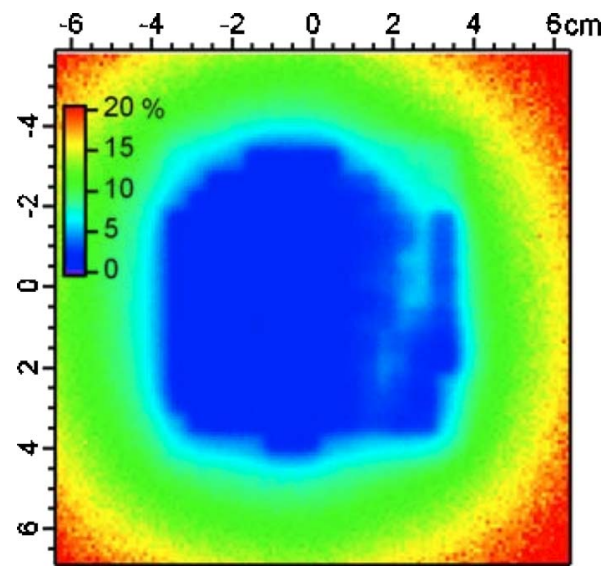

FIG. 6. The uncertainty $(100 \sigma /$ mean $)$ in the simulation of dose delivered to a static phantom. The percent relative uncertainties in dose were, on average, within $2 \%$ of the exposed region for all simulations in this study. seen in the simulations, such as tongue-and-groove effect and the interplay between leaf motion and target volume motion. These differences may be due to many factors, which include deviations in the MLC model compared to the physical design, uncertainties in spatial registration of the simulation and film, film processing and digitization, and over response of the film to lower energy scattered radiation.

\section{B. Dosimetric errors due to motion}

The magnitude and location of dosimetric errors due to motion depends on several factors. These included the amplitude and temporal characteristics of the target volume motion profile, the complexity of the fluence distribution, which determines the trajectories of each leaf pair, whether the beam is delivered with dynamic or step-and-shoot IMRT, and the ability of the MLC to deliver the leaf sequence. The largest errors (both in magnitude and in spatial extent) occur just outside the fluence pattern along the direction of motion, with lateral dimensions in the beam's eye view that are defined by the width of the beam and the range of target volume motion. The magnitude of these errors may be of any size but tend to be large due too beamlets which are preferentially optimized to create large gradients at clinically important anatomical or biological boundaries. These types of errors are shown in Fig. 4, where dose distributions in moving phantoms have been compared to those in static phantoms. Similarly, regions intended to receive low dose may move into adjacent high-fluence beamlets. The volume and magnitude of these errors tend to be lower than those outside of the fluence pattern. Likewise, regions of high dose may receive more or less dose due to motion along local dose gradients.

Parts of the target volume that are under smooth regions of the fluence pattern are typically expected to receive the expected dose regardless of motion. However, as seen in the measurements and simulations of IMRT fields delivered to phantoms under clinically measured motions (Fig. 3), such regions with low dose gradients may be delivered at different times due to differing dose modulation under adjacent leaf pairs earlier in the delivery. Consequently, temporal interplay among adjacent leaf pairs and target volume motion may create sizable dose discrepancies even in areas of a fluence 


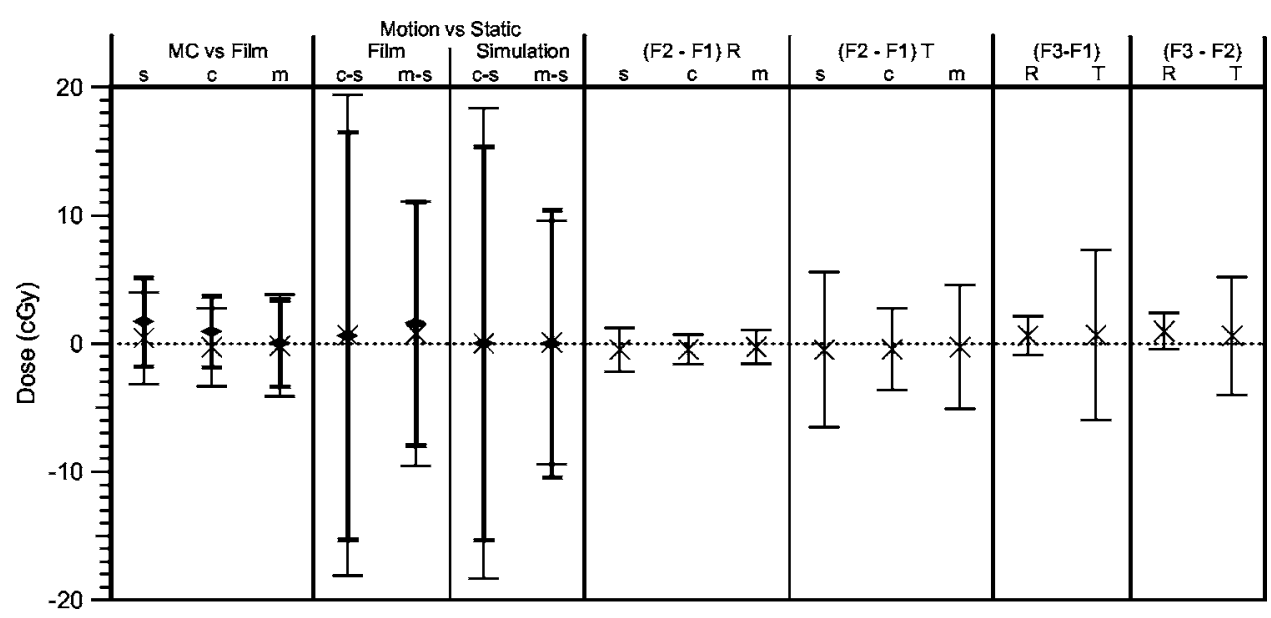

FIG. 7. Summary of average values from each column of Tables I and II. Comparisons shown with an " $X$ " represents the average dose difference for case 1 , from Table I, while data shown in bold and with diamonds represents average dose difference for case 2 , from Table II. Standard deviations $( \pm 1 \mathrm{~s})$ are represented by the error bars.

pattern with no dose gradient. Such temporal interplay effects are seen to create average hot spots of $+12 \%$ with a lateral extent of $0.5 \mathrm{~cm} \times 6 \mathrm{~cm}$ in Figs. 4(b) and 4(d) near the top of the field, with a corresponding cold spot below it. These types of dosimetric errors are not seen in the dose distributions delivered with the idealized cosine motion.

Such features of the delivered dose distribution are well reproduced by the SDDR method as characterized by the similar standard deviations Tables II and III and Fig. 7 for simulations of film measurements. Comparisons of films to simulations show standard deviations of 3-4 cGy with relatively little variation based on motion. As a base line for comparison of standard deviation values, repeat films in static and moving phantoms were measured on the same machine for all beams in case 1 . These dose distributions were registered and the differences show a standard deviation of about $1.5 \mathrm{cGy}$, while alignment with the template gives much larger standard deviations of about $5 \mathrm{cGy}$. Consequently, simulation to film agreement is seen to be better than repeat film measurements when spatially aligned with $0.1 \mathrm{~cm}$ accuracy.

\section{Dosimetric uncertainty due to system latencies}

The real-time tracking methods for the MLC and the target volume are subject to system latencies that contribute to the dosimetric uncertainty. The latency in the Varian DynaLog files is known to be about 0.05 s. ${ }^{9,45,63}$ The Calypso system makes measurements at $10 \mathrm{~Hz}$ giving a worst case latency time of $0.1 \mathrm{~s}^{64} \mathrm{In}$ our clinic, IMRT treatments are delivered at a dose rate of $400 \mathrm{MU} / \mathrm{min}$, and $1 \mathrm{MU}$ is calibrated to be $0.8 \mathrm{cGy}$ at $10 \mathrm{~cm}$ depth in $10 \times 10 \mathrm{~cm}^{2}$ field with an SSD of $90 \mathrm{~cm}$. Consequently, the dosimetric uncertainty due to system latencies may be estimated to be

$$
\Delta D \cong \frac{0.8 \mathrm{cGy}}{\mathrm{MU}}\left(\frac{400 \mathrm{MU}}{\min }\right) \frac{1 \mathrm{~min}}{60 \mathrm{~s}}(0.05+0.1) \mathrm{s}=0.8 \mathrm{cGy},
$$

which, in the worst case, is still less than $1 \%$ of the maximum dose in each beam.

\section{Time-dependent geometric patient model}

The experiments presented here were conducted in rigid, nondeforming water-equivalent phantoms where the position of each volume element was known with high positional accuracy $(d x<0.05 \mathrm{~cm})$, at any given time $(d t<0.01 \mathrm{~s})$. In clinical practice, the accuracy of this technique will be limited by the spatial and temporal accuracy of the fourdimensional (4D) patient model used in the SDDR method. The level of spatial accuracy required to realize a potential therapeutic gain might vary throughout the model, though at critical boundaries they would certainly need to be less than the planning target volume margins used in treatment planning. As new imaging and tracking technologies are introduced in the treatment room, more accurate $4 \mathrm{D}$ patient data will become available as input for this method. Similar to treatment planning methods that seek to account for motion, the simplest implementation of a 4D patient model would simply shift the planning CT based on real-time target volume tracking techniques. The next more sophisticated patient model would simply use daily CBCT data to create a deformed model of the patient that is then rigidly translated using real-time target volume tracking data. In the future, more accurate $4 \mathrm{D}$ models may be created using real-time surface tracking, fluoroscopy, and electromagnetic tracking to deform CBCT data in real time. Spatial accuracy would then likely depend on the accuracy of future deformable image mapping models or finite element modeling methods. As in-room imaging and tracking technologies evolve, along with deformable image registration methods and computer speed, it remains to be seen how accurate and practical 4D data-driven patient models become over time. In addition, to assess the clinical value of this work, the dose will have to be accumulated over the entire course of treatment and its value for modifying treatment plans and treatment delivery assessed versus the effort and cost of implementing the SDDR method at the required level of accuracy.

\section{CONCLUSIONS}

These results indicate that the synchronized dynamic dose reconstruction method is capable of combining real-time tar- 
get volume motion data and real-time machine configuration data to reproduce delivered dose distributions within the measurement and simulation uncertainties in the rigid phantom geometries considered here. The average discrepancy between film measurement and simulations is less than $1 \mathrm{cGy}$, and the large deviations created by effects such as MLC and target volume motion interplay, and tongue-andgroove effect, are reproduced spatially and dosimetrically. The standard deviations of these large differences agree also agree within $1 \mathrm{cGy}$. This is made possible through temporal synchronization of the machine fluence and motion profiles, currently available in the Varian DynaLog files, with realtime target volume motion profiles. In the future, we plan to conduct studies to correlate accumulated dose in prostate patients with clinical outcomes to verify clinical usefulness of the synchronized dynamic dose reconstruction method. As in-room image guidance and real-time tracking technologies advance in the coming years, along with deformable image mapping, this technique may prove to provide therapeutic benefit in dynamic refinement and delivery of a patient's therapy.

\section{ACKNOWLEDGMENTS}

N.T. and I.J.C. are supported in part by Grant No. R01CA106770 from the NIH (NCI). This work was also supported in part by Grant No. 1PO1CA59827-01 from the NCI.

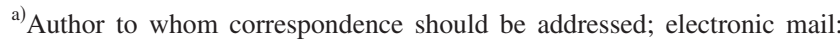
litzen@umich.edu

${ }^{1}$ C. X. Yu, D. A. Jaffray, and J. W. Wong, "The effects of intra-fraction organ motion on the delivery of dynamic intensity modulation," Phys. Med. Biol. 43(1), 91-104 (1998).

${ }^{2}$ P. J. Keall, V. R. Kini, S. S. Vedam, and R. Mohan, "Motion adaptive X-ray therapy: A feasibility study," Phys. Med. Biol. 46, 1-10 (2001).

${ }^{3}$ S. B. Jiang, C. Pope, K. M. Al Jarrah, J. H. Kung, T. Bortfeld, and G. T. Chen, "An experimental investigation on intra-fractional organ motion effects in lung IMRT treatments," Phys. Med. Biol. 48(12), 1773-1784 (2003).

${ }^{4}$ T. R. Bortfeld, D. L. Kahler, T. J. Waldron, and A. L. Boyer, "X-ray field compensation with multileaf collimators," Int. J. Radiat. Oncol., Biol., Phys. 28(3), 723-730 (1994).

${ }^{5}$ S. M. Crooks, L. F. McAven, D. F. Robinson, and L. Xing, "Minimizing delivery time and monitor units in statis IMRT by leaf-sequencing," Phys. Med. Biol. 47, 3105-3116 (2003).

${ }^{6}$ M. L. Dirkx, B. J. Heijmen, and J. P. van Santvoort, "Leaf trajectory calculation for dynamic multileaf collimation to realize optimized fluence profiles," Phys. Med. Biol. 43(5), 1171-1184 (1998).

${ }^{7}$ S. Kamath, S. Sahni, J. Palta, and S. Ranka, "Algorithms for optimal sequencing of dynamic multileaf collimators," Phys. Med. Biol. 49, 33-54 (2004).

${ }^{8}$ M. Langer, V. Thai, and L. Papiez, "Improved leaf sequencing reduces segments or monitor units needed to delivery IMRT using multileaf collimators," Med. Phys. 28(12), 2450-2458 (2001).

${ }^{9}$ D. W. Litzenberg, J. M. Moran, and B. A. Fraass, "Incorporation of realistic delivery limitations into dynamic MLC treatment delivery," Med. Phys. 29(5), 810-820 (2002).

${ }^{10}$ L. Ma, A. L. Boyer, L. Xing, and C. M. Ma, "An optimized leaf-setting algorithm for beam intensity modulation using dynamic multileaf collimators," Phys. Med. Biol. 43(6), 1629-1643 (1998).

${ }^{11}$ C. B. Saw, R. A. C. Siochi, K. M. Ayyangar, W. Zhen, and C. A. Enke, "Leaf sequencing techniques for MLC-based IMRT," Med. Dosim 26(2), 199-204 (2001).

${ }^{12}$ J. P. van Santvoort and B. J. Heijmen, "Dynamic multileaf collimation without 'tongue-and-groove' underdosage effects," Phys. Med. Biol. 41(10), 2091-2105 (1996).
${ }^{13}$ P. Xia and L. Verhey, "Multileaf collimator leaf sequencing algorithm for intensity modulated beams with multiple static segments," Med. Phys. 25(8), 1424-1434 (1998).

${ }^{14}$ Y. Yang and L. Xing, "Incorporating leaf transmission and head scatter corrections into step-and-shoot leaf sequences for IMRT," Int. J. Radiat. Oncol., Biol., Phys. 55(4), 1121-1134 (2003).

${ }^{15}$ W. Que, J. Kung, and J. Dai, “Tongue-and-groove' effect in intensity modulated radiotherapy with static multileaf collimator fields," Phys. Med. Biol. 49(3), 399-405 (2004).

${ }^{16}$ S. Kamath, S. Sahni, S. Ranka, J. Li, and J. Palta, "A comparison of step-and-shoot leaf sequencing algorithms that eliminate tongue-andgroove effects," Phys. Med. Biol. 49(14), 3137-3143 (2004).

${ }^{17}$ S. Kamath, S. Sahni, J. Palta, S. Ranka, and J. Li, "Optimal leaf sequencing with elimination of tongue-and-groove underdosage," Phys. Med. Biol. 49(3), 7-19 (2004).

${ }^{18}$ D. Jianrong and W. Que, "Simultaneous minimization of leaf travel distance and tongue-and-groove effect for segmental intensity-modulated radiation therapy," Phys. Med. Biol. 49(23), 5319-5331 (2004).

${ }^{19}$ F. Haryanto, M. Fippel, A. Bakai, and F. Nusslin, "Study on the tongue and groove effect of the Elekta multileaf collimator using Monte Carlo simulation and film dosimetry," Strahlenther. Onkol. 180(1), 57-61 (2004).

${ }^{20}$ T. LoSasso, C.-S. Chui, and C. C. Ling, "Comprehensive quality assurance for the delivery of intensity modulated radiotherapy with a multileaf collimator used in the dynamic mode," Med. Phys. 28(11), 2209-2219 (2001).

${ }^{21}$ G. A. Ezzell and S. Chungbin, "The overshoot phenomenon in step-andshoot IMRT delivery," J. Appl. Clin. Med. Phys. 2(3), 138-148 (2001).

${ }^{22}$ P. Xia, C. F. Chuang, and L. J. Verhey, "Communication and sampling rate limitations in IMRT delivery with a dynamic multileaf collimator system," Med. Phys. 29(3), 412-423 (2002).

${ }^{23}$ L. Ma, P. B. Geis, and A. L. Boyer, "Quality assurance for dynamic multileaf collimator modulated fields using a fast beam imaging system," Med. Phys. 24(8), 1213-1220 (1997).

${ }^{24}$ J. Sonke, J. M. Balter, M. van Herk, L. Zijp, M. L. Kessler, and R. Kashani, "Recalculation of dose changes due to breathing movement assessed from respiratory-correlated cone beam CT," Int. J. Radiat. Oncol., Biol., Phys. 60(s1), S289 (2004).

${ }^{25}$ B. Schaly, G. S. Bauman, J. J. Battista, and J. V. Dyk, "Validation of contour-driven thin-plate splines for tracking fraction-to-fraction changes in anatomy and radiation therapy dose mapping," Phys. Med. Biol. 50, 459-475 (2005).

${ }^{26}$ J. M. Balter, K. L. Lam, C. J. McGinn, T. S. Lawrence, and R. K. Ten Haken, "Improvement of CT-based treatment-planning models of abdominal targets using static exhale imaging," Int. J. Radiat. Oncol., Biol., Phys. 41(4), 939-943 (1998).

${ }^{27}$ I. J. Chetty, M. Rosu, D. L. McShan, B. A. Fraass, J. M. Balter, and R. K. Ten Haken, "Accounting for center-of-mass target motion using convolution methods in Monte Carlo-based dose calculations of the lung," Med. Phys. 31(4), 925-932 (2004).

${ }^{28}$ I. J. Chetty, M. Rosu, N. Tyagi, L. H. Marsh, D. L. McShan, J. M. Balter, B. A. Fraass, and R. K. Ten Haken, "A fluence convolution method to account for respiratory motion in three-dimensional dose calculations of the liver: A Monte Carlo study," Med. Phys. 30(7), 1776-1780 (2003).

${ }^{29}$ P. Giraud, Y. De Rycke, B. Dubray, S. Helfre, D. Voican, L. Guo, J. C. Rosenwald, K. Keraudy, M. Housset, E. Touboul, and J. M. Cosset, "Conformal radiotherapy (CRT) planning for lung cancer: Analysis of intrathoracic organ motion during extreme phases of breathing," Int. J. Radiat. Oncol., Biol., Phys. 51(4), 1081-1092 (2001).

${ }^{30}$ P. J. Keall, J. V. Siebers, S. Joshi, and R. Mohan, "Monte Carlo as a four-dimensional radiotherapy treatment-planning tool to account for respiratory motion," Phys. Med. Biol. 49(16), 3639-3648 (2004).

${ }^{31} \mathrm{~J}$. Li and L. Xing, "Inverse planning incorporating organ motion," Med. Phys. 27(7), 1573-1578 (2000).

${ }^{32}$ D. M. Lockman, D. Yan, and J. Wong, "Estimating the dose variation in a volume of interest with explicit consideration of patient geometric variation," Med. Phys. 27(9), 2100-2108 (2000).

${ }^{33}$ A. E. Lujan, J. M. Balter, and R. K. Ten Haken, "A method for incorporating organ motion due to breathing into $3 \mathrm{D}$ dose calculations in the liver: Sensitivity to variations in motion," Med. Phys. 30(10), 2643-2649 (2003).

${ }^{34}$ T. Bortfeld, K. Jokivarsi, M. Goitein, J. Kung, and S. B. Jiang, "Effects of intra-fraction motion on IMRT dose delivery: Statistical analysis and 
simulation," Phys. Med. Biol. 47(13), 2203-2220 (2002).

${ }^{35}$ I. J. Chetty, K. L. Lam, J. M. Balter, R. K. Ten Haken, D. L. McShan, and H. M. Sandler, "Dosimetric implications of approximating setup uncertainties with Gaussian distributions in Monte Carlo based prostate cancer treatment planning," Radiother. Oncol. (submitted) (2005).

${ }^{36}$ I. J. Chetty, M. Rosu, D. L. McShan, B. A. Fraass, and R. K. Ten Haken, "Inverse plan optimization incorporating random setup uncertainties using Monte Carlo based fluence convolution,” Int. J. Radiat. Oncol., Biol., Phys. (submitted) (2005).

${ }^{37}$ T. R. Mackie, J. W. Scrimger, and J. J. Battista, "A convolution method of calculating dose for 15 MV x rays," Med. Phys. 12(2), 188-196 (1985).

${ }^{38}$ R. George, S. S. Vedam, T. D. Chung, V. Ramakrishnan, and P. J. Keall, "The application of the sinusoidal model to lung cancer patient respiratory motion," Med. Phys. 32(9), 2850-2861 (2005).

${ }^{39} \mathrm{~S}$. Webb, "The effect on IMRT conformality of elastic tissue movement and a practical suggestion for movement compensation via the modified dynamic multileaf collimator (dMLC) technique," Phys. Med. Biol. 50(6), 1163-1190 (2005).

${ }^{40} \mathrm{~S}$. Webb, "Motion effects in (intensity modulated) radiation therapy: A review," Phys. Med. Biol. 51(13), R403-R425 (2006).

${ }^{41}$ L. Papiez, D. Rangaraj, and P. Keall, "Real-time DMLC IMRT delivery for mobile and deforming targets," Med. Phys. 32(9), 3037-3048 (2005).

${ }^{42}$ C. S. Chui, E. Yorke, and L. Hong, "The effects of intra-fraction organ motion on the delivery of intensity-modulated field with a multileaf collimator," Med. Phys. 30(7), 1736-1746 (2003).

${ }^{43}$ S. A. Naqvi and W. D. D'Souza, "A stochastic convolution/superposition method with isocenter sampling to evaluate intrafraction motion effects in IMRT," Med. Phys. 32(4), 1156-1163 (2005).

${ }^{44}$ D. W. Litzenberg, J. M. Moran, and B. A. Fraass, "Verification of dynamic and segmental IMRT delivery by dynamic log file analysis," J. Appl. Clin. Med. Phys. 3(2), 63-72 (2002).

${ }^{45}$ A. M. Stell, J. G. Li, O. A. Zeidan, and J. F. Dempsey, "An extensive log-file analysis of step-and-shoot intensity modulated radiation therapy segment delivery errors," Med. Phys. 31(6), 1593-1602 (2004).

${ }^{46}$ J. G. Li, J. F. Dempsey, L. Ding, C. Liu, and J. R. Palta, "Validation of dynamic MLC-controller log files using a two-dimensional diode array," Med. Phys. 30(5), 799-805 (2003).

${ }^{47}$ O. A. Zeidan, J. G. Li, M. Ranade, A. M. Snell, and J. F. Dempsey, "Verification of step-and-shoot IMRT delivery using a fast video-based electronic portal imaging device," Med. Phys. 31(3), 463-476 (2004).

${ }^{48}$ P. Zygmanski, J. H. Kung, S. B. Jiang, and L. Chin, "Dependence of fluence errors in dynamic IMRT on leaf-positional errors varying with time and leaf number," Med. Phys. 30(10), 2736-2749 (2003).

${ }^{49}$ J. M. Balter, S. Hadley, D. Litzenberg, H. Sandler, E. Vertatschitsch, S. Dimmer, T. Willoughby, and P. Kupelian, "Accuracy of a 4D magnetic localization system: Evaluation in the clinical environment," Int. J. Radiat. Oncol., Biol., Phys. 60(1 Supplement), S612-S613 (2004).

${ }^{50}$ T. Willoughby, P. Kupelian, J. Pouliot, K. Shinohara, M. Aubin, M. RoachIII, L. Skrumeda, J. Balter, D. Litzenberg, S. Hadley, J. Wei, and H. Sandler, "Implant experience and positional stability of AC magnetic bea$\mathrm{con}(\mathrm{tm})$ transponders used to localize patients for external beam radiation therapy of the prostate," Int. J. Radiat. Oncol., Biol., Phys. 60(Suppl 1),
S267-S268 (2004).

${ }^{51}$ D. W. Litzenberg, J. M. Balter, S. W. Hadley, H. M. Sandler, T. R. Willoughby, P. A. Kupelian, and L. Levine, "The influence of intrafraction motion on margins for prostate radiotherapy," Int. J. Radiat. Oncol., Biol., Phys. 65(2), 548-553 (2006).

${ }^{52}$ J. Sempau, S. J. Wilderman, and A. F. Bielajew, "DPM, a fast, accurate Monte Carlo code optimized for photon and electron radiotherapy treatment planning dose calculations," Phys. Med. Biol. 24, 2263-2291 (2000).

${ }^{53}$ I. J. Chetty, P. M. Charland, N. Tyagi, D. L. McShan, B. A. Fraass, and A. F. Bielajew, "Photon beam relative dose validation of the DPM Monte Carlo code in lung-equivalent media," Med. Phys. 30(4), 563-573 (2003).

${ }^{54}$ I. J. Chetty, J. J. DeMarco, and T. D. Solberg, "A virtual source model for Monte Carlo modeling of arbitrary intensity distributions," Med. Phys. 27(1), 166-172 (2000).

${ }^{55}$ I. J. Chetty, J. M. Moran, D. L. McShan, B. A. Fraass, S. J. Wilderman, and A. F. Bielajew, "Benchmarking of the Dose Planning Method (DPM) Monte Carlo code using electron beams from a racetrack microtron," Med. Phys. 29(6), 1035-1041 (2002).

${ }^{56}$ N. Tyagi, I. J. Chetty, B. A. Fraass, and A. F. Bielajew, "Calculations of a Millennium Multileaf Collimator using the DPM and BEAM/DOSXYZ Monte Carlo codes," Med. Phys. 29(6), 1230 (2002).

${ }^{57}$ I. J. Chetty, N. Tyagi, M. Rosu, P. M. Charland, D. L. McShan, R. K. Ten Haken, B. A. Fraass, and A. F. Bielajew, presented at the Nuclear Mathematical and Computational Sciences: A Century in Review, A Century Anew, Gatlinburg, TN, 2003 (unpublished).

${ }^{58}$ M. Rosu, M. M. Coselmon, E. Acosta, B. Fraass, M. D, and I. J. Chetty, "Implementation and initial testing of a Monte Carlo based algorithm for IMRT inverse treatment planning," Med. Phys. (accepted).

${ }^{59}$ N. Tyagi, J. M. Moran, D. W. Litzenberg, A. F. Bielajew, B. A. Fraass, and I. J. Chetty, "Experimental verification of a Monte Carlo-based source model for IMRT treatment planning," Med. Phys. (accepted).

${ }^{60}$ D. W. O. Rogers, B. R. Walters, and I. Kawrakow, BEAMnrc Users Manual: National Research Council Report PIRS-0509(A) rev. H. (Ottawa, Canada, 2004).

${ }^{61}$ D. W. Litzenberg, S. W. Hadley, K. L. Lam, and J. M. Balter, "A precision translation stage for reproducing measured target volume motions," J. Appl. Clin. Med. Phys. (accepted).

${ }^{62} \mathrm{P}$. Thévenaz and M. Unser, "A pyramid approach to subpixel registration based on intensity," IEEE Trans. Image Process. 7, 27-41 (1998).

${ }^{63}$ C. Burman, C. S. Chui, G. Kutcher, S. Leibel, M. Zelefsky, T. LoSasso, S. Spirou, Q. Wu, J. Yang, J. Stein, R. Mohan, Z. Fuks, and C. C. Ling, "Planning delivery and quality assurance of intensity-modulated radiotherapy using dynamic multileaf collimator: A strategy for large-scale implementation for the treatment of carcinoma of the prostate," Int. J. Radiat. Oncol., Biol., Phys. 39(4), 863-873 (1997).

${ }^{64}$ J. M. Balter, J. N. Wright, L. J. Newell, B. Frieme, S. Dimmer, Y. Cheng, J. Wong, E. Vertatschitsch, and T. P. Mate, "Accuracy of a wireless localization system for radiotherapy," Int. J. Radiat. Oncol., Biol., Phys. 61(3), 933-937 (2005). 